\title{
Automatic termination of parallel optimization runs of stochastic global optimization methods in consensus or stagnation cases
}

\author{
Jurijs Sulins ${ }^{1,2 *}$, Martins Mednis ${ }^{1,2}$ \\ ${ }^{1}$ Biosystems Group, Department of Computer Systems, Latvia University of Agriculture, Liela iela 2, LV-3001, Jelgava, Latvia \\ ${ }^{2}$ SIA TIBIT, Rīgas iela 52, LV-3018, Ozolnieki, Ozolnieku nov., Latvia \\ * Corresponding author \\ jurijs.sulins@hotmail.com
}

Received: 8 May 2012; accepted: 19 May 2012; published online: 21 May 2012

This paper has no supplementary material.

\begin{abstract}
Dynamic models give detailed information about the influence of many parameters on the behaviour of the biochemical process of interest. Parameter optimization of dynamic models is used in parameter estimation tasks and in design tasks. A drawback of the popular family of global stochastic optimization methods is the stochastic nature of the convergence of the best value of objective function to the global optimum or a value close to that. Therefore the optimization can take long time until a stable value of objective function is reached. Even then the risk of stagnation far from global optimum remains. That sets force to look for efficient approaches to reduce optimization time and discover cases of poor performance of optimization methods.

Parallel optimization runs of identical optimization tasks can be used to reduce the impact of stochastic processes used in stochastic optimization methods. Consensus and stagnation criteria are proposed to terminate a set of parallel optimization runs when it is assessed that no significant improvements of the best value of the objective function are expected.

Four automatically detectable cases of behaviour of a group of parallel optimization runs are analysed: 1) reaching of consensus criterion (consensus case), 2) stagnation of all optimization runs without reaching the consensus criterion (stagnation case), 3) stagnation at the initial value of the objective function, 4) lack of feasible solution.

The proposed approach can be used automating the termination of optimization process when no further progress of the best value of objective function is expected. Suitability of particular optimization method with its settings for particular optimization task can be assessed analysing the dynamics of objective function's best values of parallel runs.
\end{abstract}

Keywords: optimization, parameter estimation, design task, dynamic modelling, convergence dynamics.

\section{Introduction}

The mission of systems biology and synthetic biology in metabolic engineering tasks (Mendes and Kell, 1998) is to facilitate the development of new bioprocesses by the help of in silico procedures thus reducing the amount of necessary biological experiments which are more costly both in terms of time and resources.

Dynamic models give detailed information about the influence of many parameters of the network like kinetic parameters of reactions and concentrations of reactants (Stelling, 2004). The most typical approach to represent biochemical networks is through a set of coupled deterministic ordinary differential equations intended to describe the network and the production and consumption rates for the individual species involved in the network (Balsa-Canto et al., 2010). The expected increase of the size of dynamic models (Jamshidi and Palsson, 2008) will facilitate their application. Serious challenge in case of optimization of dynamic model is lack of analytical optimization solutions to solve systems of nonlinear differential equations.

Therefore the numerical methods are used in optimization tasks of biochemical networks. The numerical methods can be classified as local and global optimum seeking methods (Balsa-Canto et al., 2008; Mendes and Kell, 1998). Usually the global optimization methods are used to avoid stagnation of the solution in local optima. There are two classes of global numerical optimization methods: deterministic and stochastic. The advantage of some of deterministic methods is the guaranteed reach of global optimum for the price of unknown computation time (Banga, 2008; Moles et al., 2003). Therefore, the stochastic global optimization methods are the most popular in optimization tasks of biochemical networks due to their universality and relatively fast convergence to the global optimum close value (Banga, 2008; Moles et al., 2003).

In case of single optimization run of stochastic global optimization method the termination criterion usually is a stable best value of the objective function for a relatively long time and it cannot be determined if that is a stagnation at local optima or the best value is reached. Therefore in case of stagnation of a single optimization run at local optima misleading conclusions can be done about the optimization potential of given set of adjustable parameters (Mozga and Stalidzans, 2011c).

The convergence of global stochastic optimization methods is analysed in case of parameter estimation tasks (Baker et al., 2010; Balsa-Canto et al., 2008, 2010; Mendes and Kell, 1998; Moles et al., 2003). Convergence dynamics for design optimization (Mendes and Kell, 1998) or more generally process optimization tasks where the properties of metabolic pathways are changed with the aim of enhancing the 
production of some metabolite of interest (Mendes and Kell, 1998; Moles et al., 2003) is analyzed in several recent publications (Mozga and Stalidzans, 2011b, 2011c; Mozga et al., 2011). A software tool ConvAn (Kostromins et al., 2012) for analysis of convergence dynamics suitable for both parameter estimation and design tasks has been developed for statistical analysis of performance of stochastic optimization methods. The convergence speed and reliability of optimization method are critical in design problems of biochemical networks (Mozga and Stalidzans, 2011b, 2011c) where even relatively small number (5-15) of adjustable parameters of the model cause hundreds or thousands of combinations to be explored (Mozga and Stalidzans, 2011a). The combinatorial explosion of number of adjustable parameter combinations sets force to look for efficient approaches to reduce necessary optimization time.

A set of criteria is proposed to terminate a parallel optimization runs when it is assessed that no significant improvements of the best value of objective function are expected. The first criterion is the consensus of parallel optimization runs which indicate that all the parallel optimization runs have converged via different trajectories to the same solution indicating also good performance of the optimization method (Mozga and Stalidzans, 2011b, 2011c). The second criterion is a long stagnation of all optimization runs at different best values indicating poor performance of optimization method (Mozga and Stalidzans, 2011b, 2011c; Mozga et al., 2011).

Use of proposed criteria for automatic termination of optimization both for parameter estimation and design tasks reduce the length of optimization experiment by more intensive use of computational resources due to parallel optimization runs. The main advantage compared to a single optimization run is the early detection of the best value (consensus of independent optimization runs) or bad performance of optimization (stagnation of at least one optimization run).

\section{Materials and methods}

Yeast glycolysis models from Biomodels data base (Le Novère et al., 2006) are used to examine the performance of consensus and stagnation criteria. Criteria are demonstrated in design optimization tasks where objective function has to be maximized. Software COPASI (Hoops et al., 2006) is used as optimization tool. Parallel optimization experiments using stochastic global optimization methods with COPASI 4.7 Build 34 are automatically set and performed using software CoRunner (Sulins and Stalidzans, 2012). Since stochastic optimization methods involve use of random numbers, successive optimization runs on the same model with the same algorithm converge to the best value in a different trajectory. Convergence dynamics of optimization runs is analysed using software ConvAn (Kostromins et al., 2012).

In the maximization experiments the values are normalized the way that $0 \%$ of objective function value corresponds to the objective function value of unmodified model while $100 \%$ correspond to the best value of objective function found in any of parallel runs in particular time moment. Thus the value of objective function that correspond $0 \%$ remains constant while the value of $100 \%$ increases during optimization until the best value is reached or stagnation starts.
In case of minimization experiments the best value of objective function is decreasing and the module of changes of the best value of objective function has to be taken into account calculating $100 \%$ value.

Consensus criterion is fulfilled when all of parallel optimization runs reach a value of objective function which lies within pre-defined consensus corridor. The consensus corridor can be expressed in per cents: $3 \%$ corridor would mean that the best values of all parallel optimization runs have to be within $97-100 \%$ corridor. Criterion was analysed optimizing yeast glycolysis model of Galazzo and Bailey (Galazzo and Bailey, 1990) for ethanol production (RodríguezAcosta et al., 1999). The model contains 2 compartments, 8 reactions and 9 metabolites. Objective function in all optimization runs was to maximize flux of pyruvate kinase which is proportional to the ethanol production. Concentrations of enzymes catalysing reactions ATPase, GAP, Glucose in, Hexokinase, Phosphofructokinase and Pyruvate kinase were chosen as adjustable parameters.

Evolutionary programming optimization method (Back and Schwefel, 1993; Back et al., 1997; Fogel et al., 1992) was used with following method settings: Number of Generations: 30000; Population Size: 20; Random Number Generator: 1; Seed: 0 . The values of adjustable parameters were allowed to change within a wide range from $-99 \%$ up to $900 \%$ from their initial values. "Steady state" subtask of optimization within COPASI was chosen to avoid solutions without steady state.

Stagnation criterion is fulfilled when all the parallel optimization runs do not change their best value of objective function for a pre-set stagnation delay time while the consensus is not reached. The pre-set stagnation delay time can be defined in time units or as per cents of optimization duration. Stagnation was analysed using yeast glycolysis model of Hynne and co-workers (Hynne et al., 2001). The model contains 2 compartments, 24 reactions and 25 metabolites. Objective function in all optimization runs was

$$
K=\frac{\text { Ethanol flow }}{\text { Glucose uptake }}+5 \times \text { Ethanol flow }
$$

The sets of adjustable parameters and the optimization method were chosen on purpose to observe the stagnation behaviour (Mozga and Stalidzans, 2011b). Concentrations of enzymes catalysing five reactions (Hexokinase, Alcohol dehydrogenase, ATP consumption, Glycerol synthesis, Phosphofructokinase) were chosen as adjustable parameters. Evolutionary programming optimization method (Back and Schwefel, 1993; Back et al., 1997; Fogel et al., 1992) was used with following method settings: Number of Generations: 30000; Population Size: 20; Random Number Generator: 1; Seed: 0 . The values of adjustable parameters were allowed to change within a wide range from $-99 \%$ up to $1000 \%$ from their initial values. "Steady state" subtask of optimization within COPASI was chosen to avoid solutions without steady state.

Five optimization experiments were performed for each experimental setup number of reactions for each optimization method on a server running 64-bit Microsoft Windows Server 2008 Standard Service Pack 2 operating system. Server has 4x QuadCore Intel Xeon MP E7330 2400 MHz CPU and 32768 MB of RAM. Single processor per task was used as COPASI does not support optimization with parallel task distribution. 


\section{Results and discussion}

Two criteria of termination of parallel optimization runs are tested for their ability to terminate the optimization when no significant increase of the best value is expected.

Generally there are four cases of behaviour of a group of parallel optimization runs: 1) reaching of consensus criteria (consensus case), 2) stagnation of all optimization runs without reaching the consensus criteria (stagnation case), 3) consensus at the initial value of objective function, 4) lack of feasible solution.

\subsection{Consensus}

Convergence to consensus best value of the objective function indicates good performance of optimization when all the parallel runs of stochastic optimization method have reached the same or very similar best value within the consensus corridor. That is a good reason to conclude that the best value found is close to the global optimum still keeping in mind that finding global optimum cannot be guaranteed by stochastic global optimization methods (Banga, 2008; Moles et al., 2003). A consensus delay time (determined in time units or per cents of duration of optimization runs) can be used optionally to avoid coincidental short-time consensus.

Illustrative consensus experiment (Fig.1) demonstrates application of consensus criterion. In this particular case there is no further improvement after fulfilling of automatic consensus criterion. On the other hand it is not guaranteed that there will not be further improvement as the behaviour of stochastic optimization methods cannot be predicted with full confidence. To increase the confidence about correctness of automatically made decision the number of parallel runs can be increased or the consensus corridor can be narrowed. Both changes will increase the probability of longer duration of optimization.

\subsection{Stagnation}

Stagnation case means that all the parallel optimization runs do not change their value for the delay time and at least one optimization run stagnate at value which is not within the pre-set consensus corridor of the best one gives indication about risk that the optimization method does not perform well for particular optimization task. There is increased risk that also the other runs stagnate at values which are far from the optimal solution. It is suggested to test another optimization method or settings of the method to improve the performance. In case if several methods perform similar way it might indicate the peculiarity of the model or particular set of adjustable parameters (Mozga and Stalidzans, 2011c).

There is a risk of false detection of stagnation if the pre-set stagnation delay time is too short. This kind of risk can be reduced by increased delay time which increases the duration of optimization as a consequence.
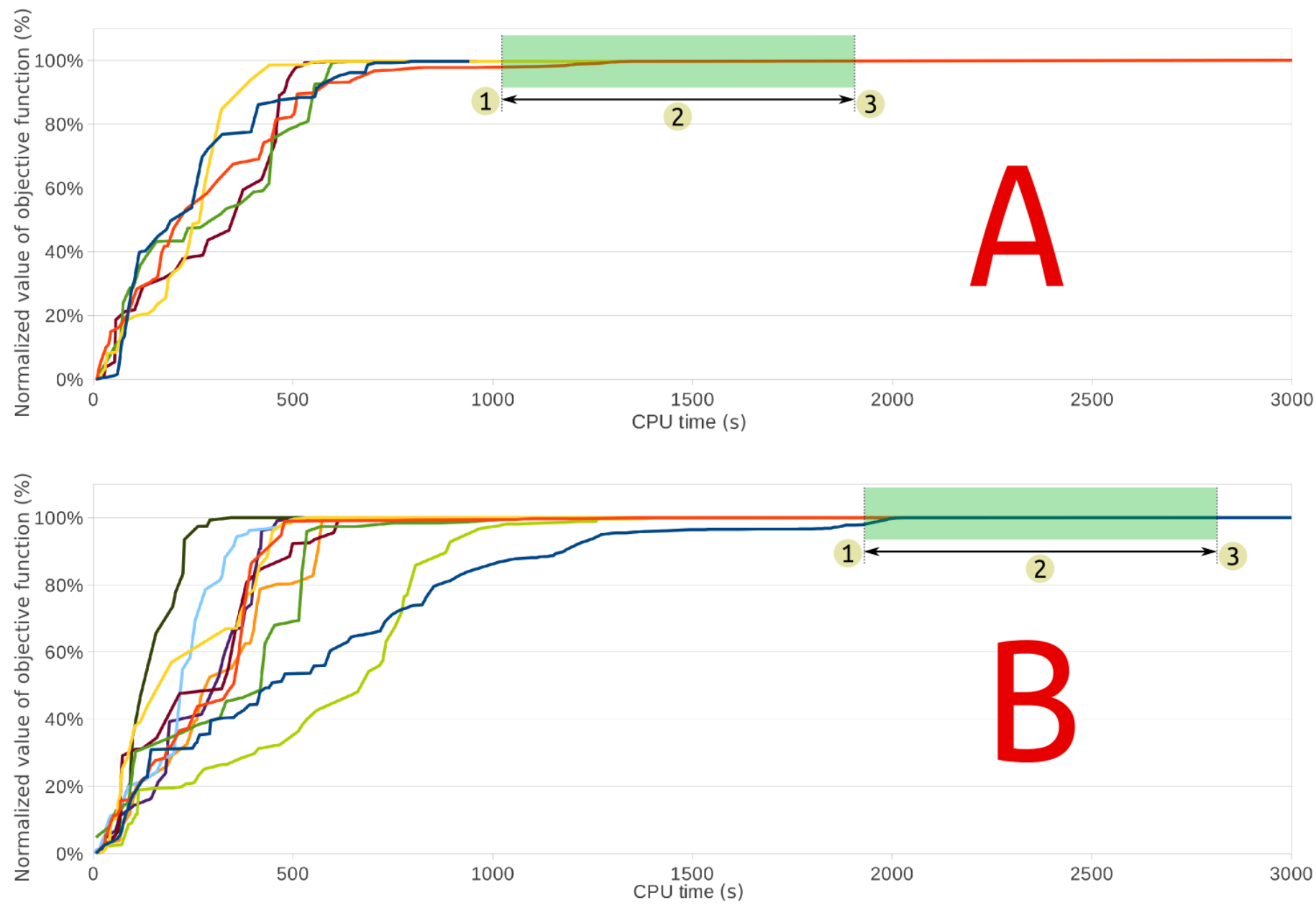

Fig. 1. The convergence dynamics of consensus case with five (a) and ten (b) parallel optimization runs. All optimization runs have reached the $3 \%$ consensus corridor in the time moment " 1 ". The consensus delay time " 2 " is $900 \mathrm{~s}$ and lasts till the termination at the time moment "3". 


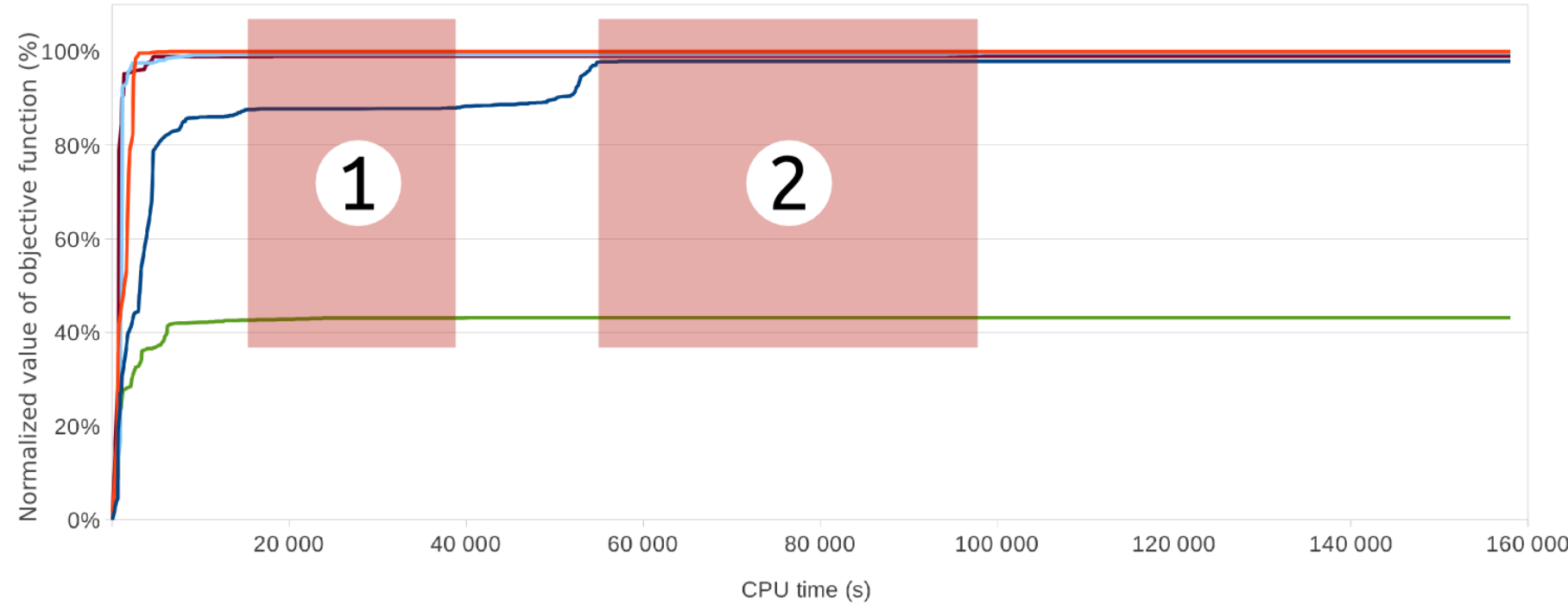

Fig. 2. The convergence dynamics of stagnation case with five parallel optimization runs. The stagnation termination criterion is fulfilled at the end of the time period " 2 " which represents the stagnation delay time. In case of a shorter stagnation time (period " 1 ") the stagnation delay time counter is reset until the next stagnation starts.

\subsection{Stagnation at the initial value}

Stagnation of all parallel runs at the initial value of the objective function can be explained at least in two ways: 1) initial parameters of the model correspond to the best parameter values within the solution space and the optimization task is completed or 2) poor performance of optimization. The first case has very low probability. Still it cannot be fully ignored being a special case of consensus. Usually stagnation at the initial value of objective function is caused by poor performance of optimization method, huge solution space due to high number of adjustable parameters, complexity of computation because of the size or peculiarities of the model or other reasons. Improvement of optimization performance can be done by alterations of optimization method or its settings. Stagnation of all parallel runs at the initial value of the objective function is interesting as formally both consensus and stagnation criteria are reached. Therefore it is necessary to test if the value of objective function of initial model is improved to recognize this case automatically. Optimization can be terminated if initial value is not improved by any of parallel runs for some delay time.

\subsection{Lack of feasible solution}

Lack of feasible solution is a different case of stagnation at the initial value of objective function described above. Even very fast and reliably converging optimization method cannot find any solution if that is excluded by too strict or even contradicting constraints. In this case the best value usually is replaced by different expressions like "-INF", "NAN" or others in different optimization software. In this case it is useful first to check the existence of feasible steady states of the model with given constraints. If the feasible solution is not excluded by constraints, the optimization methods or their settings should be changed to improve the performance.

Stagnation criterion can detect this case automatically if the expression of objective function that corresponds to the lack of any solution with steady state in particular optimization tool is known. Automatic detection of this case should be used introducing some delay time to ensure even a small feasible area in the proposed solution space to be found.

\section{Conclusion}

Consensus and stagnation criteria of termination of parallel optimization runs of global stochastic optimization methods have been tested for their use to terminate the optimization when no significant increase of the best value of the objective function is expected. This approach can give faster and more accurate conclusion about the best value of objective function at the cost of computational resources needed for performance of parallel runs.

Consensus criterion is fulfilled when all of parallel optimization runs reach a value of objective function which lies within pre-defined consensus corridor.

Stagnation criterion is fulfilled when all the parallel optimization runs do not change their best value of objective function for a pre-set stagnation delay time while the consensus is not reached. The pre-set stagnation delay time can be defined in time units or as per cents of optimization duration.

Generally there are four automatically detectable cases of behaviour of a group of parallel optimization runs: 1) reaching of consensus criterion (consensus case), 2) stagnation of all optimization runs without reaching the consensus criterion (stagnation case), 3) stagnation at the initial value of the objective function, 4) lack of feasible solution. Optimization task can be considered as successfully completed only in the consensus case. Still also the other cases give valuable information about reasons of failure of particular setting of optimization task or optimization methods.

To reduce the risk of finding suboptimal solution the number of parallel runs can be increased or the consensus corridor can be narrowed. The side effect is the increase of probability of longer optimization duration. The probability of false detection of stagnation can be reduced by increase of the pre-set delay time causing increase of optimization duration.

\section{References}

Back, T. and Schwefel, H.P. (1993), "An Overview of Evolutionary Algorithms for Parameter Optimization" Evolutionary Computation, Vol. 1 No. 1, pp. 1-23. http://dx.doi.org/10.1162/evco.1993.1.1.1

Back, T., Fogel, D.B., Michalewicz, Z. (1997), Handbook of Evolutionary Computation, Oxford: IOP Publishing/Oxford University Press, p.1130. http://dx.doi.org/10.1887/0750308958 
Baker, S.M., Schallau, K., Junker, B.H. (2010), “Comparison of different algorithms for simultaneous estimation of multiple parameters in kinetic metabolic models" Journal of integrative bioinformatics, Vol. 7 No. 3, pp. 1-9. http://dx.doi.org/10.2390/biecoll-jib-2010-133

Balsa-Canto, E., Alonso, A., Banga, J.R. (2010), "An iterative identification procedure for dynamic modeling of biochemical networks" BMC systems biology, Vol. 4, 11. http://dx.doi.org/10.1186/1752-0509-4-11

Balsa-Canto, E., Peifer, M., Banga, J.R., Timmer, J., Fleck, C. (2008), "Hybrid optimization method with general switching strategy for parameter estimation" BMC systems biology, Vol. 2, 26. http://dx.doi.org/10.1186/1752-0509-2-26

Banga, J.R. (2008), "Optimization in computational systems biology" BMC systems biology, Vol. 2, 47. http://dx.doi.org/10.1186/1752-0509-2-47

Fogel, D.B., Fogel, L.J., Atmar, J.W. (1992), "Meta-evolutionary programming" in Proceedings of 25th Asiloma Conference on Signals, Systems and Computers, Asilomar, pp. 540-545.

Galazzo, J.L., and Bailey, J.E. (1990), "Fermentation pathway kinetics and metabolic flux control in suspended and immobilized Saccharomyces cerevisiae" Enzyme and Microbial Technology, Vol. 12 No. 3, pp. 162172. http://dx.doi.org/10.1016/0141-0229(90)90033-M

Hoops, S., Sahle, S., Gauges, R., Lee, C., Pahle, J., Simus, N., Singhal, M., et al. (2006), "COPASI - a COmplex PAthway Simulator" Bioinformatics (Oxford, England), Vol. 22 No. 24, pp. 3067-3074. http://dx.doi.org/10.1093/bioinformatics/btl485

Hynne, F., Danø, S., Sørensen, P.G. (2001), "Full-scale model of glycolysis in Saccharomyces cerevisiae" Biophysical chemistry, Vol. 94 No. 1-2, pp. 121-63. http://dx.doi.org/10.1016/S0301-4622(01)00229-0

Jamshidi, N., and Palsson, B.Ø. (2008), "Formulating genome-scale kinetic models in the post-genome era" Molecular systems biology, Vol. 4, 171. http://dx.doi.org/10.1038/msb.2008.8

Kostromins, A., Mozga, I., Stalidzans, E. (2012), "ConvAn: a convergence analyzing tool for optimization of biochemical networks" Biosystems, Vol. 108 Nr. 1-3, pp. 73-77. http://dx.doi.org/10.1016/j.biosystems.2011.12.004

Le Novère, N., Bornstein, B.J., Broicher, A., Courtot, M., Donizelli, M., Dharuri, H., Li, L., et al. (2006), "BioModels Database: a free, centralized database of curated, published, quantitative kinetic models of biochemical and cellular systems" Nucleic acids research, Vol. 34 No. Database issue, D689-691. http://dx.doi.org/10.1093/nar/gkj092

Mendes, P., and Kell, D.B. (1998), "Non-linear optimization of biochemical pathways: applications to metabolic engineering and parameter estimation" Bioinformatics (Oxford, England), Vol.14 No.10, pp. 869-83. http://dx.doi.org/10.1093/bioinformatics/14.10.869

Moles, C.G., Mendes, P., Banga, J.R. (2003), "Parameter estimation in biochemical pathways: a comparison of global optimization methods" in Skjoldebremd, C., Trystrom, G. (Eds.), Genome Research, Vol. 13 No. 11, pp. 2467-2474. http://dx.doi.org/10.1101/gr.1262503

Mozga, I, and Stalidzans, E. (2011a), "Optimization protocol of biochemical networks for effective collaboration between industry representatives, biologists and modellers" Proceedings of International Industrial Simulation Conference, 6-8 June 2011, Venice, pp. 91-96.

Mozga, I, and Stalidzans, E. (2011b), "Convergence Dynamics of Biochemical Models To The Global Optimum" Proceedings of 3rd International Conference on E-Health and Bioengineering, 24-26 November 2011, Iasi, pp. 227-230.

Mozga, I, and Stalidzans, E. (2011c), "Convergence dynamics of biochemical pathway steady state stochastic global optimization" Proceedings of IEEE 12th International Symposium on Computational Intelligence and Informatics (CINTI), 21-22 November 2011, Budapest, pp. 231-235. http://dx.doi.org/10.1109/CINTI.2011.6108504

Mozga, I, Kostromins, A., Stalidzans, E. (2011) "Forecast of Numerical Optimization Progress of Biochemical Networks" Proceedings of International conference on Engineering for Rural Development, 26-27 May 2011, Jelgava, pp. 103-108.

Rodríguez-Acosta, F., Regalado, C.M., Torres, N.V. (1999), "Non-linear optimization of biotechnological processes by stochastic algorithms: application to the maximization of the production rate of ethanol, glycerol and carbohydrates by Saccharomyces cerevisiae" Journal of biotechnology, Vol. 68 No. 1, pp. 15-28. http://dx.doi.org/10.1016/S01681656(98)00178-3

Stelling, J. (2004), "Mathematical models in microbial systems biology" Current opinion in microbiology, Vol. 7, No. 5, pp. 513-518. http://dx.doi.org/10.1016/j.mib.2004.08.004

Sulins, J., and Stalidzans, E. (2012), "CoRunner: multiple optimization run manager for COPASI software" Proceedings of International conference on Applied Information and Communication Technologies, 26-27 April 2012, Jelgava, pp. 312 - 316. 